\title{
Textile-based Radio Frequency Energy Harvesting and Storage using Ultra-Compact Rectennas with High Effective-to-Physical Area Ratio
}

\author{
$1^{\text {st }}$ Mahmoud Wagih \\ Electronics and Computer Science \\ University of Southampton \\ Southampton, U.K. \\ mahm1g15@ecs.soton.ac.uk \\ $4^{\text {th }}$ Steve Beeby \\ Electronics and Computer Science \\ University of Southampton \\ Southampton, U.K. \\ spb@ecs.soton.ac.uk
}

\author{
$2^{\text {nd }}$ Nicholas Hillier \\ Electronics and Computer Science \\ University of Southampton \\ Southampton, U.K. \\ nh1g09@soton.ac.uk
}

\author{
$3^{\text {rd }}$ Alex S. Weddell \\ Electronics and Computer Science \\ University of Southampton \\ Southampton, U.K. \\ asw@ecs.soton.ac.uk
}

\begin{abstract}
Wearable Radio Frequency (RF) rectennas do not require expensive or hazardous materials and can be easily integrated with conventional e-textiles. In this paper, we investigate the use of ultra-miniaturized wire-type monopole antennas for energy harvesting (EH) applications, as a method maximizing the effective collection area of a rectenna relative to its physical size, while not reducing the net DC output. The rectenna, operating in the $915 \mathrm{MHz}$ band, is integrated with a simple carbon-based e-textile supercapacitor for direct energy conversion and storage. The integrated module is then demonstrated, for the first time, wirelessly-charging a Bluetooth Low Energy sensor node at over $1 \mathrm{~m}$ distance from a license-free Powercast transmitter. The $\mathbf{1 4 . 1} \mathrm{mF}$ supercapacitor is charged using the e-textile rectenna filament in $83 \mathrm{~s}$ up to $4.14 \mathrm{~V}$, from an incident power density of $23.9 \mu \mathrm{W} / \mathrm{cm}^{2}$ and a timeaveraged efficiency over $40 \%$, enabling the sensor node to sustain operation for $108 \mathrm{~s}$ after the wireless RF source is stopped. Compared to state-of-the-art RF energy harvesters, the proposed module achieves over five fold improvement in the RF to DC power harvesting efficiency normalized to the harvester's area.

Index Terms-Antenna, energy harvesting, rectifier, rectenna, supercapacitor, wireless power transmission, wireless sensor nodes.
\end{abstract}

\section{INTRODUCTION}

Powering wearable devices using radio frequency (RF) energy harvesting and wireless power transmission (WPT) has attracted significant research interest due to the simple construction of rectennas and their compatibility with different e-textile fabrication methods [1]-[4]. Textile-based rectennas have been realized using electro-plated conductive fabrics and threads [1], [2], [5], [6], screen-printed inks [3], and thin and flexible polyimide copper laminates [4], [7], enabling

This work was supported by the UK Engineering and Physical Sciences Research Council (EPSRC) under Grants EP/P010164/1 and EP/L016818/1, and the European Commission under the EnABLES Project, grant: 730957. operation up to millimeter-wave frequencies for operating in future $5 \mathrm{G}+$ bands [8].

It is widely known that the DC output of energy harvesters, from an available power density, scales with the size of the harvester [9]. This has been widely explored for large and miniaturized vibrational energy harvesters and solar cells [9]. In the same sense, antennas need to be comparable to the wavelength of operation to achieve a high radiation efficiency and gain, subsequently increasing their effective area [10]. Large-area non-wearable [11], and textile-based wearable RF energy harvesters composed of several rectenna elements [3], [5] as well as electrically-large patch antennas [2] have been reported. Moreover, several studies have investigated rectenna miniaturization, presenting rectennas as small as $0.06 \lambda^{2}$ [12]. Nevertheless, the sensitivity of such implementations has been lower than state-of-the-art high-efficiency textile-based rectifiers [13], which limits their suitability for harvesting $\mu \mathrm{W} / \mathrm{cm}^{2}$ power densities efficiently. Furthermore, while a compact rectenna was integrated with a textile supercapacitor in [7], none of the previously-reported far-field e-textile rectennas was demonstrated powering a real load such as a duty-cycled sensor node.

In this paper, we investigate the relation between the physical area/RF EH by using a miniaturized wire-type meandered monopole to directly charge a two-cell e-textile supercapacitor which is used to power a wireless sensor node. It is demonstrated that the physical area of an RF EH antenna can be reduced by over five fold, without reducing the effective area, allowing for a very high output DC power density. The realized rectenna is used to charge an e-textile supercapacitor and is demonstrated, for the first time, powering a real wireless sensor node load, based on an Arm microcontroller unit (MCU) and a Bluetooth Low Energy (BLE) transceiver 


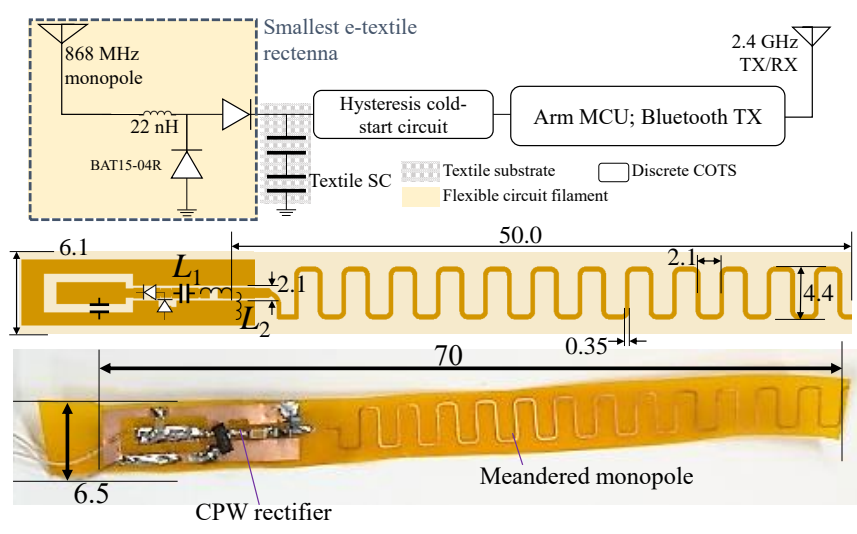

Fig. 1. Schematic, layout (dimensions in $\mathrm{mm}$ ), and photograph of the miniaturized flexible filament RF EH system.

system on chip (SoC). Section II presents the componentlevel design and characterization. In Section III, the RF charging of the textile supercapacitor and the wireless node is demonstrated and compared to state-of-the-art RF harvesters.

\section{System Design AND Simulation}

The proposed system is composed of a flexible e-textile rectenna, a textile carbon-based supercapacitor, and an Armbased BLE SoC, as shown in Fig. 1. To fabricate a thin and flexible textile-friendly rectenna, a single-layered structure is needed. As a result, a coplanar waveguide (CPW) antenna and rectifier designs are adopted. Distributed elements matching is avoided to maintain compactness where lumped inductors will not degrade the power conversion efficiency [13].

\section{A. Antenna Design and Characterization}

To begin with, a CPW monopole antenna, with a length $=\lambda / 4$ at $915 \mathrm{MHz}$, was designed for benchmarking the meander-line monopole's RF performance. The compact rectenna design is then achieved through ground miniaturization and meandering the monopole radiator. Meander-line monopoles are widely used where miniaturized antennas are required and were found to be suitable for wearable applications through length tuning [7]. By reducing the ground plane size, the antenna's overall size can be significantly reduced without affecting its effective collection area, which is predominantly determined by the antenna's radiation efficiency for an antenna harvesting arbitrarily-directed radiation [14].

Both the standard $\lambda / 4$ monopole and the meander-line monopole with a compact ground were simulated in CST Microwave Studio, to observe their gain and subsequently EH area. Fig. 2 shows the simulated realized gain and total efficiency of the meandered monopole and the straight $\lambda / 4$ monopole. Based on the simulated gain, it can be seen that despite the meandered monopole occupying $13 \%$ of its standard $\lambda / 4$ counterpart's area, it maintains a comparable gain and total efficiency which implies a similar EH area.

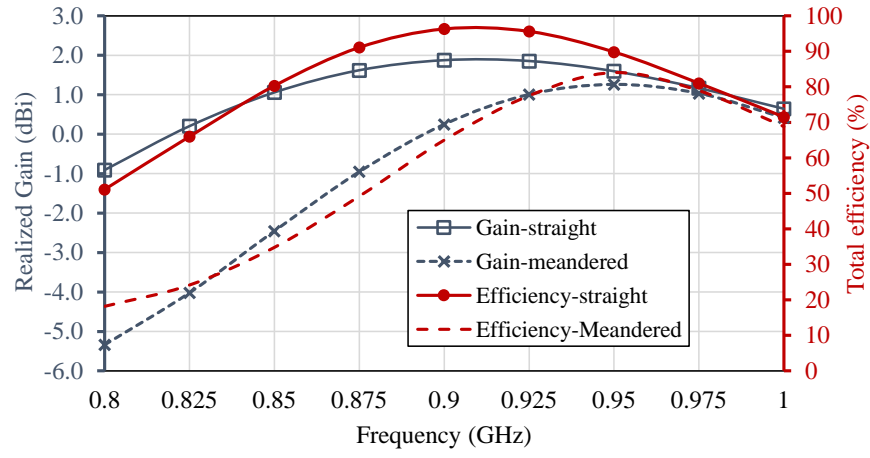

Fig. 2. Simulated realized gain and total efficiency of a benchmark $35 \mathrm{~cm}^{2}$ standard $\lambda / 4$ monopole and the $4.55 \mathrm{~cm}^{2}$ meander-line monopole.

To explain, the effective collection area $A_{\text {eff. }}$ of a receiving antenna is given by

$$
A_{\text {eff. }}=G \frac{\lambda^{2}}{4 \pi},
$$

where $G$ is the antenna's realized gain [10]. Based on their simulated gains, the effective-to-physical size ratio of the proposed meandered monopole and the conventional $\lambda / 4$ monopole is 23.3 and 3.9 , respectively. Therefore, the use of highly miniaturized antennas in compact devices has the potential for significant performance improvement over conventional antenna designs of larger physical size.

\section{B. Rectifier Design and Characterization}

As the antenna is matched to a $50 \Omega$ characteristic impedance, the rectifier's input matching needs to be designed to match the same standard. By selecting a $50 \Omega$ antenna/rectifier design, a wider range of conventional antenna designs can be adopted in the integrated rectenna [15]. The rectifier is based on a compact CPW layout, to minimize the insertion losses and maintain overall compactness, and is matched using a $22 \mathrm{nH}$ lumped wirewound inductor $\left(L_{1}\right)$. As shown in Fig. 1, $L_{2}$ is added to provide a DC current return path and to improve the rectenna's matching.

The rectifier's PCE was characterized using a $50 \Omega$ signal source directly connected to its input. Fig. 3 shows the simulated measured DC output of the rectifier, across a $5 \mathrm{k} \Omega$ load, determined following a resistive load sweep to find the optimum load impedance. Observing the $8 \mathrm{~V}$ peak DC output, high PCE, and relatively low optimum load impedance of $5 \mathrm{k} \Omega$, the rectifier can be used to directly charge a supercapacitor without a standalone power management circuit or DC-DC converters, further reducing the size and complexity of the complete system.

\section{Supercapacitor Fabrication and Characterization}

The supercapacitor used in this work is based on a plain cotton substrate. The carbon electrode ink was produced from $0.85 \mathrm{~g}$ of carbon (9:1 ratio of activated carbon to carbon black), $0.15 \mathrm{~g}$ ethylene-vinyl acetate and $5 \mathrm{ml}$ of 1,2,4-trichlorobenzene. The ink was then spray deposited 


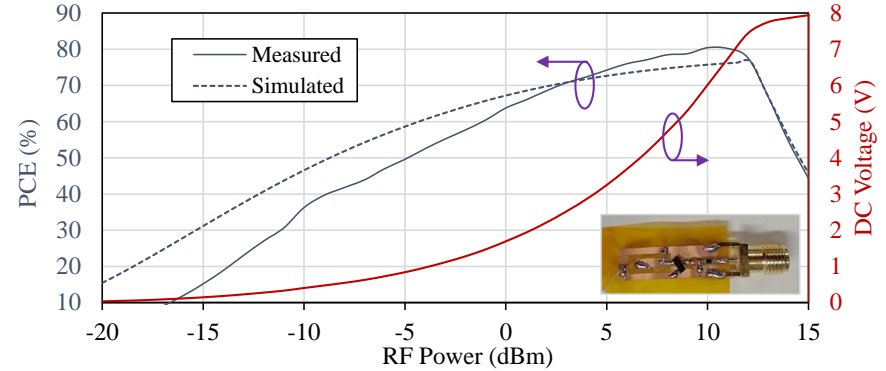

Fig. 3. Simulated (dashed) and measured (solid) PCE and DC voltage output of the rectifier for a $5 \mathrm{k} \Omega$ fixed load.

onto either side of the cotton, forming a single layered device. These devices were subsequently impreganted with a $0.5 \mathrm{M}$ tetraethylammonium tetrafluoroborate/polyacrylamide electrolyte [16].

As the supercapacitor can only operate up to $2.4 \mathrm{~V}$, two cells are connected in series to enable operation up to $4 \mathrm{~V}$. By reaching a higher DC potential, the additional energy stored in the supercapacitor enables the load to operate for a prolonged time period, as well as withstand the high start-up current (momentarily reaching $20 \mathrm{~mA}$ ) of the BLE SoC. At a discharge current of $0.196 \mathrm{~mA}$, the two-cell supercapacitor module had a capacitance of $14.1 \mathrm{mF}$, characterized via galvanostatic cycling. The devices maintained a capacitance of $11.3 \mathrm{mF}$ up to a measurement current of $2.37 \mathrm{~mA}$.

\section{RECTENNA RFEH CHARACTERIZATION}

The integrated system is characterized using a wireless power source to evaluate its end-to-end efficiency, as well as compare its performance to state-of-the-art textile and compact RF energy harvesters. A $915 \mathrm{MHz} 3 \mathrm{~W}$ licensefree Powercast source is used to power the rectenna at $1 \mathrm{~m}$, resulting in an incident power density of $23.9 \mu \mathrm{W} / \mathrm{cm}^{2}$. The rectenna is directly connected to the supercapacitor, where the DC output is defined as the net energy stored in the supercapacitor. Unlike recently reported rectennas [1]-[3], this includes the losses in the energy storage device as well as the power conversion losses between the rectifier and the varying impedance of the supercapacior [7]. Furthermore, by quantifying the performance of the rectenna in terms of the net stored energy, the efficiency is calculated based on the net useful energy which can be directly used to power offthe-shelf systems.

For an RF-charged capacitor, the time-averaged PCE is

$$
\mathrm{PCE}_{\text {Average }}=\frac{C V^{2}}{2} \times \frac{1}{t} \times \frac{1}{P_{\mathrm{RF}}},
$$

where $C$ is the capacitance of the $14.1 \mathrm{mF}$ textile supercapacitor, $V$ is the peak DC potential, $t$ is the charging time taken to reach $\approx 4.1 \mathrm{~V}$, and $P_{\mathrm{RF}}$ is the RF power collected by the antenna, excluding impedance mismatch and rectifier losses [13]. Therefore, all losses in the system, e.g. capacitor leakage, rectifier losses, and impedance mismatch between the rectifier and the antenna, are factored in $\mathrm{PCE}_{\text {Average }}$.
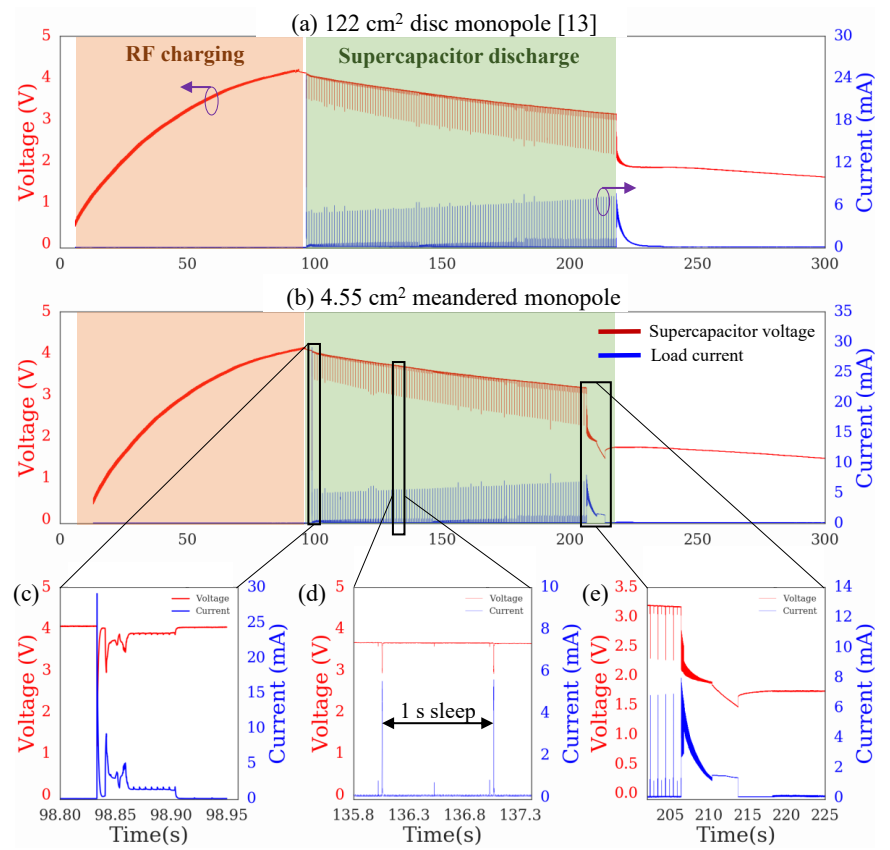

Fig. 4. Measured DC output of the rectenna across the textile supercapacitor and the current drawn by the load post-charging: (a) large-area disc monopole [13]; (b) the miniaturized monopole; (c) the capacitor voltage and current draw during start-up; (d) two duty-cycled BLE advertisement packets happening at a $1 \mathrm{~s}$ period; (e) MCU shutdown when $V_{C}$ decays under $1.8 \mathrm{~V}$.

TABLE I

DC OUTPUT OF THE COMPACT AND CONVENTIONAL MONOPOLES

\begin{tabular}{|l|l|l|l|l|}
\hline & Area $\left(\mathrm{cm}^{2}\right)$ & $t(\mathrm{~s})$ & PCE Average & PHE \\
\hline \hline $\begin{array}{l}\text { Disc } \\
\text { Monopole }\end{array}$ & 124 & 87 & $\begin{array}{l}44.77 \\
( \pm 5 \%)\end{array}$ & 0.47 \\
\hline $\begin{array}{l}\text { Meandered } \\
\text { filament }\end{array}$ & 4.55 & 83 & $45.5( \pm 5 \%)$ & 13.2 \\
\hline
\end{tabular}

For the purpose of demonstrating a functional far-field wirelessly-powered system, the cold-start circuit and the MCU shown in Fig. 1 were implemented on a standard PCB. Nevertheless, the system could also be implemented on a flexible substrate as demonstrated in [17], where a BLE transceiver was integrated with an EH power management circuit and a wearable antenna on a flexible substrate.

Fig. 4 shows the charging curve of the supercapacitor for both a $10 \times 10 \mathrm{~cm}^{2}$ broadband monopole antenna [13], [18], and the $7 \times 0.6 \mathrm{~cm}^{2}$ meander-line monopole. The operation of the Bluetooth wireless sensor node is demonstrated through the current draw measured through a current-sense resistor. The DC output of both rectennas is summarized in Table I.

The proposed harvester is compared to state-of-the-art textile-based and compact rectennas. The Figure of Merit (FoM), in this case the Power Harvesting Efficiency (PHE) relative to the antenna's physical size is defined as

$$
\mathrm{PHE}=\frac{P_{\mathrm{DC}}}{S A_{\text {Physical }}}=\frac{C V^{2}}{2} \times \frac{1}{t} \times \frac{1}{S A_{\text {Physical }}},
$$

where $P_{\mathrm{DC}}$ is the rectenna's DC output, $S$ is the incident RF power density, and $A_{\text {Physical }}$ is the antenna's area. This 


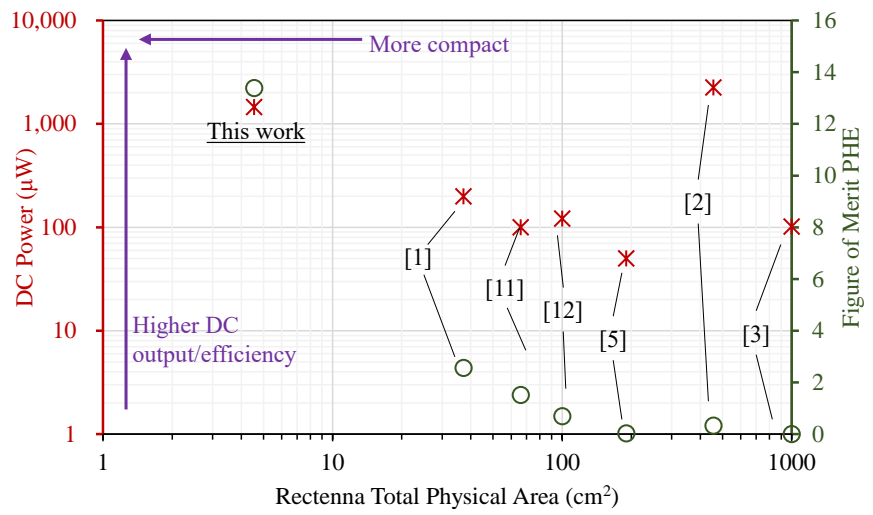

Fig. 5. Comparison of the proposed rectenna with recent implementations.

definition enables holistic evaluation of the rectenna which includes the antenna's losses and aperture efficiency [3].

Observing Table I and Fig. 4, it can be seen that despite the compact meandered monopole and the broadband monopole having significantly different physical areas, they both harvest approximately the same amount of energy in a comparable time due to having a comparable gain. Noting that the meandered monopole occupies over $95 \%$ smaller area, it can be concluded that miniaturizing the RF EH antenna, without reducing its effective collection area, does not decrease the EH efficiency or net system output. Using (3), it can be seen in Table I that the compact meandered filament achieves a significantly higher PHE, when normalizing its PCE to the harvester's physical area.

The performance of the compact meandered monopole, in terms of DC power output and PHE, calculated using (3), is compared to recently reported textile-based rectennas, in Fig. 5. The DC power output is presented for the maximum reported $S$, at which the rectenna's PCE plateaus.

Comparing [2], operating at a similar frequency and power density, the patch antenna, whose gain is higher than the wiretype dipole generates a higher DC output. However, when the antenna's size is considered, the PHE FoM reduces for larger antenna due to its lower aperture efficiency. Therefore, for rectennas where directional radiation patterns, [2], or a broad bandwidth [3], [13] are not needed, miniaturized wire-type antennas will generate the highest PHE, relative to their physical size. Furthermore, while the gain, and subsequently $A_{\mathrm{Eff}}$, of the antenna will decrease by up to $10 \mathrm{~dB}$ in proximity with the human body [3], [13], the proposed EH and storage module can be used to charge the antenna intermittently away from the human body, as well as in other applications such as asset tracking of e-textiles.

\section{CONCLUSION}

In this paper, the performance of highly-miniaturized flexible rectennas for textile-integrated RF EH was investigated, showing a five fold improvement in the total harvested energy relative to the harvester area compared to state-ofthe-art rectennas. By designing RF energy harvesters based on miniaturized yet high radiation efficiency meandered wiretype antennas, it is possible to minimize the device's physical footprint without reducing its effective collection area. The integrated rectenna/supercapacitor module was demonstrated experimentally powering a BLE Arm MCU for $108 \mathrm{~s}$, following $83 \mathrm{~s}$ of charging at a power density of $23.9 \mu \mathrm{W} / \mathrm{cm}^{2}$, received at $1 \mathrm{~m}$ from a license-free Powercast transmitter.

\section{REFERENCES}

[1] M. Wagih, G. S. Hilton, A. S. Weddell, and S. Beeby, "Dual-Band DualMode Textile Antenna/Rectenna for Simultaneous Wireless Information and Power Transfer (SWIPT)," IEEE Trans. Antennas Propag., 2021.

[2] G. Monti, L. Corchia, and L. Tarricone, "UHF Wearable Rectenna on Textile Materials," IEEE Trans. Antennas. Propag., vol. 61, 7, pp. 3869 - 3873, 2013.

[3] J. A. Estrada, E. Kwiatkowski, A. López-Yela, M. Borgoñós-García, D. Segovia-Vargas, T. Barton, and Z. Popović, "An RF-Harvesting Tightly-Coupled Rectenna Array Tee-Shirt with Greater than Octave Bandwidth," IEEE Trans. Microw. Theory Techniq., vol. 68 no. 9, pp. 3908 - 3919, 2020.

[4] M. Wagih, G. S. Hilton, A. S. Weddell, and S. Beeby, "Broadband Millimetre-Wave Textile-based Flexible Rectenna for Wearable Energy Harvesting ," IEEE Trans. Microw Theory Techn, vol. 68 no. 11, pp. 4960 - 4972, 2020.

[5] D. Vital, S. Bhardwaj, and J. L. Volakis, "Textile Based Large Area RF-Power Harvesting System for Wearable Applications," IEEE Trans. Antennas Propag., vol. 68, no. 3, pp. 2323 - 2331, 2019.

[6] M. Wagih, G. S. Hilton, A. S. Weddell, and S. Beeby, "Dual-polarized wearable antenna/rectenna for full-duplex and mimo simultaneous wireless information and power transfer (swipt)," IEEE Open Journal of Antennas and Propagation, vol. 2, pp. 844-857, 2021.

[7] M. Wagih, N. Hillier, S. Yong, A. S. Weddell, and S. Beeby, "Rfpowered wearable energy harvesting and storage module based on etextile coplanar waveguide rectenna and supercapacitor," IEEE Open Journal of Antennas and Propagation, vol. 2, pp. 302 - 314, 2021.

[8] M. Wagih, A. S. Weddell, and S. Beeby, "Millimeter-Wave Power Harvesting: A Review," IEEE Open Journal of Antennas and Propagation, vol. 1 , pp. 560 - 578, 2020.

[9] P. D. Mitcheson, E. M. Yeatman, G. K. Rao, A. S. Holmes, and T. C. Green, "Energy harvesting from human and machine motion for wireless electronic devices," Proceedings of the IEEE, vol. 96, no. 9, pp. 1457-1486, 2008.

[10] C. A. Balanis, "Antenna Theory: Analysis and Design. Third Edition," Wiley Interscience, pp. $84-85,2005$.

[11] Z. Popovic, S. Korhummel, S. Dunbar, R. Scheeler, A. Dolgov, R. Zane, E. Falkenstein, and J. Hagerty, "Scalable rf energy harvesting," IEEE Transactions on Microwave Theory and Techniques, vol. 62, no. 4, pp. 1046-1056, 2014.

[12] A. Okba, A. Takacs, and H. Aubert, "Compact rectennas for ultra-lowpower wireless transmission applications," IEEE Trans. Microw. Theory Techn., vol. 67, 5, pp. $1697-1707,2019$.

[13] M. Wagih, A. S. Weddell, and S. Beeby, "Omnidirectional DualPolarized Low-Profile Textile Rectenna with over $50 \%$ Efficiency for Sub- $\mu \mathrm{W} / \mathrm{cm}^{2}$ Wearable Power Harvesting," IEEE Transactions on Antennas and Propagation, vol. 69, no. 5, pp. 2522-2536, 2021.

[14] S. Shen, Y. Zhang, C.-Y. Chiu, and R. Murch, "An Ambient RF Energy Harvesting System Where the Number of Antenna Ports Is Dependent on Frequency," IEEE Trans. Microw. Theory Techn., vol. 67 no. 9, pp. $3821-3832,2019$.

[15] M. Wagih, A. S. Weddell, and S. Beeby, "Rectennas for RF Energy Harvesting and Wireless Power Transfer: a Review of Antenna Design [Antenna Applications Corner]," IEEE Antennas Propag. Mag., vol. 62 no. 5 , pp. $95-107,2020$.

[16] N. Hillier, S. Yong, A. Cruden, and S. Beeby, "Acetonitrile-free organic electrolyte for textile supercapacitor applications," Journal of The Electrochemical Society, 2021.

[17] M. Wagih, Y. Wei, and S. Beeby, "Flexible $2.4 \mathrm{GHz}$ Sensor Node for Body Area Networks with a Compact High-Gain Planar Antenna," IEEE Antennas Wireless Propag. Lett., vol. 17, 12, pp. 49 - 53, 2018.

[18] J. Liang, C. Chiau, X. Chen, and C. Parini, "Study of a printed circular disc monopole antenna for uwb systems," IEEE Trans. Antennas Propag., vol. 53, no. 11, pp. 3500 - 3504, 2005. 